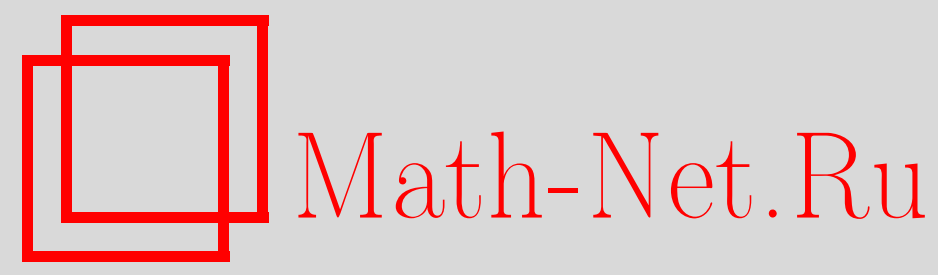

H.-J. Engelbert, J. Wolf, Strong Markov local Dirichlet processes and stochastic differential equations, Теория вероятн. и ее примен., 1998, том 43, выпуск 2, 331-348

DOI: https://doi.org/10.4213/tvp1468

Использование Общероссийского математического портала Math-Net.Ru подразумевает, что вы прочитали и согласны с пользовательским соглашением http://www . mathnet.ru/rus/agreement

Параметры загрузки:

IP: 52.90 .164 .192

26 апреля 2023 г., $17: 56: 40$

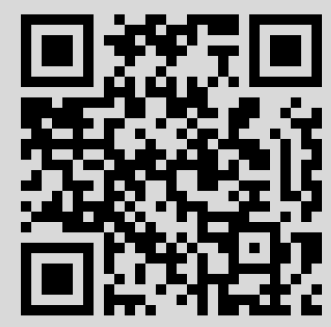


(C) $1998 \mathrm{r}$.

ENGELBERT H.-J. ${ }^{*}$, WOLF $J .^{*}$

\title{
STRONG MARKOV LOCAL DIRICHLET PROCESSES AND STOCHASTIC DIFFERENTIAL EQUATIONS
}

\begin{abstract}
Устанавливаются необходимые и достаточные условия, наложенные на естественную шкалу и меру скорости непрерывного строго марковского локального процесса Дирихле, для того, чтобы процесс имел представление в виде решения некоторого стохастического дифференциального уравнения. Результаты применяются к случаю процессов Бесселя произвольной размерности.
\end{abstract}

Ключевые слова и фразы: процессы Бесселя, процессы Дирихле, стохастические дифференциальные уравнения, локальное время, строго марковские процессы.

\section{Introduction}

K. Itô [15], [16], [17] introduced ordinary stochastic differential equations of the type

$$
X_{t}^{i}=x_{i}+\sum_{j=1}^{r} \int_{0}^{t} \sigma_{i j}\left(X_{s}\right) d B_{s}^{j}+\int_{0}^{t} b_{i}\left(X_{s}\right) d s, \quad 0 \leqslant t<\infty, 1 \leqslant i \leqslant d,
$$

where $B$ is a Brownian motion in $\mathbf{R}^{r}$ and $b_{i}, \sigma_{i j}: \mathbf{R}^{d} \rightarrow \mathbf{R}, 1 \leqslant i \leqslant d, 1 \leqslant$ $j \leqslant r$. Nowadays, solutions to such equations are called Itô diffusions. Itô's work opened a new probabilistic approach to the investigation of Markov processes and initiated a long series of interacting research activities in the fields of Markov processes and stochastic differential equations. For detailed information we refer to the book by Stroock and Varadhan [32] as well as to articles by Skorohod [31], Çinlar et al. [8], Çinlar and Jacod [9], Méléard [21] and Engelbert and Schmidt [10], [11], [12].

Chinlar and Jacod [9] provided evidence that, indeed, stochastic differential equations allow to study a great class of Markov processes. They showed that every semimartingale Hunt process can be obtained from some Itô diffusion by time change.

In the one-dimensional case that we will exclusively deal with throughout this paper local time gives rise to a somewhat more general class of

*Friedrich-Schiller-Universität, Fakultät für Mathematik und Informatik, Institut für Stochastik, 07740 Jena, Germany. 
stochastic differential equations, the so-called stochastic differential equations with generalized drift

$$
X_{t}=X_{0}+\int_{0}^{t} b\left(X_{s}\right) d B_{s}+\int_{\mathbf{R}} L^{X}(t, y) v(d y), \quad t \geqslant 0,
$$

where $B$ is a Brownian motion, $L^{X}$ denotes the local time of the solution, $b$ a measurable function and $v$ a signed measure. Stochastic differential equations with generalized drift were studied, among others, by Portenko [23], Perkins [22], Le Gall [19], [20] and Engelbert and Schmidt [10], [12]. We point out that the solutions to the equations (1) and (2) are always semimartingales, but do not necessarily enjoy the Markov property. However, strong Markov solutions play a key role since every solution is obtained from a strong Markov one by time change (see [12]).

So having seen stochastic differential equations to admit strong Markov solutions we are led to the converse problem to find out whether or not a given strong Markov process is a solution to a stochastic differential equation. Engelbert and Schmidt (see [12], [29], [30]) derived necessary and sufficient conditions for a continuous strong Markov semimartingale to solve the stochastic differential equations (1) and (2), respectively, in terms of the scale function and the speed measure. Let us recall that these quantities uniquely determine a Markov process.

In recent years, not least thanks to the theory of Dirichlet forms, the class of local Dirichlet processes has been gaining importance. It extends the class of semimartingales and, although the theorem of Bichteler and Dellacherie (see [24, Theorem III.22]) characterizes semimartingales as the only «good» integrators, admits elements of stochastic analysis (see [2], [3], [4], [13], [14], [27], [28], [34], [35]). Let us recall that a continuous local Dirichlet process is defined to be a process which can be written as the sum of a continuous local martingale and a continuous adapted process of zero quadratic variation. Using methods of stochastic analysis, especially the generalized Bouleau-Yor formula (see [34, 2.2]), the second author of the present paper, showed in [36] that, under weak assumptions, a continuous strong Markov local Dirichlet process $X$ takes the form

$$
\begin{aligned}
X_{t}= & X_{0}+M_{t}+d_{+}\left(X_{0}, \int_{0}^{t} 1_{S_{+}}\left(X_{s}\right) d s\right)-d_{-}\left(X_{0}, \int_{0}^{t} 1_{S_{-}}\left(X_{s}\right) d s\right) \\
& -\frac{1}{2} \int\left(p^{-1}\right)^{\prime}(y) \mathbf{1}_{p(R)}(y) d_{y} L^{p(X)}(t, y),
\end{aligned}
$$

where $M$ is a continuous local martingale, $R$ denotes the set of regular points of $X, S_{+}$and $S_{-}$are certain subsets of the right singular and left singular points, respectively, $d_{ \pm}$are deterministic functions and $p$ is a scale function. The integral with respect to the semimartingale local time $L^{p(X)}$ is defined in the sense of Bouleau and Yor (see [34]). 
Let us recall that Bouleau and Yor [7] introduced the stochastic integral of the type $\int f(a) d_{a} L^{Z}(t, a)$, where $f$ is a locally bounded function and $Z$ a semimartingale, as a unique continuous extension of the mapping given on step functions by

$$
f=\sum_{i=1}^{n} f_{i} \mathbf{1}_{\left(a_{i}, a_{i+1}\right]} \mapsto \sum_{i=1}^{n} f_{i}\left(L^{Z}\left(t, a_{i+1}\right)-L^{Z}\left(t, a_{i}\right)\right)=: f(a) d_{a} L^{Z}(t, a) .
$$

Thereby, they established a change of variables formula.

In view of the representation (3), the problem if the continuous strong Markov local Dirichlet process $X$ is a solution to a stochastic differential equation amounts to asking if the local martingale part can be written as a stochastic integral $M_{t}=\int_{0}^{t} \sigma\left(X_{s}\right) d B_{s}$ for some Brownian motion $B$.

In this paper we will derive necessary and sufficient conditions on the scale function and the speed measure of $X$ ensuring that $X$ satisfies

$$
\begin{aligned}
X_{t}= & X_{0}+\int_{0}^{t} \sigma\left(X_{s}\right) d B_{s}+d_{+}\left(X_{0}, \int_{0}^{t} 1_{S_{+}}\left(X_{s}\right) d s\right) \\
& -d_{-}\left(X_{0}, \int_{0}^{t} 1_{S_{-}}\left(X_{s}\right) d s\right)-\frac{1}{2} \int\left(p^{-1}\right)^{\prime}(y) 1_{p(R)}(y) d_{y} L^{p(X)}(t, y) .
\end{aligned}
$$

Furthermore, we will compute the coefficient $\sigma$. We will apply these results to Bessel processes, thus obtaining a new stochastic differential equation in dimensions $0<d<1$ and recovering the known equations in dimensions $d \geqslant 1$.

Thus, starting with the analysis of strong Markov processes, in a natural way, we are led to the stochastic differential equation (4) which describes local Dirichlet processes. We remark that, if the solution is a semimartingale, (4) coincides with the stochastic differential equation of [1, Theorem 7.9] which is of type (2). This is seen by integration by parts $[34,5.1]$ exactly in the same way as in [36, Remark 4.6].

In a subsequent paper we will study stochastic differential equations of type (4) replacing the local time $L^{p(X)}$ of the transformed process by the local time of $X$ itself. We will derive conditions for existence and uniqueness and look for the strong Markov property of solutions (see also [33, Chapter 2]).

\section{Definitions and prerequisits}

Let $I$ denote an interval of $\mathbf{R}, \mathscr{B}(I)$ the $\sigma$-algebra of Borel subsets of $I$ and $\mathscr{B}^{u}(I)$ its universal completion. We consider a family $\left(\Omega, \mathscr{F}, \mathbf{P}_{x} ; x \in\right.$ I) of probability spaces such that $\left(\mathbf{P}_{x}\right)_{x \in \mathbf{R}}$ is a probability kernel from $\left(I, \mathscr{B}^{u}(I)\right)$ into $(\Omega, \mathscr{F})$. For every probability measure $\mu$ on $(I, \mathscr{B}(I))$ we define a probability measure $\mathrm{P}_{\mu}$ on $(\Omega, \mathscr{F})$ via

$$
\mathbf{P}_{\mu}(A):=\int_{\mathbf{R}} \mathbf{P}_{x}(A) \mu(d x), \quad A \in \mathscr{F} .
$$


Replacing $\mathscr{F}$ by its completion with respect to the family $\left(\mathbf{P}_{\mu}\right)$ and extending $\mathbf{P}_{\mu}$ in the natural way we may assume $\mathscr{F}$ to be complete with respect to $\left(\mathbf{P}_{\mu}\right)$. Furthermore, let $\mathbf{F}=\left(\mathscr{F}_{t}\right)_{t \geqslant 0}$ denote a right-continuous filtration with $\mathscr{F}_{t} \subseteq$ $\mathscr{F}$ such that $\mathscr{F}_{0}$ (and consequently every $\mathscr{F}_{t}$ ) is complete with respect to $\left(\mathbf{P}_{\mu}\right)$. We write $\left(\Omega, \mathscr{F}, \mathbf{F}, \mathbf{P}_{x} ; x \in I\right)$ for the family of filtered probability spaces. We say that an assertion holds a.s. if it holds $\mathbf{P}_{x^{-}}$a.s. for every $x \in I$.

If $X$ is a process on $\left(\Omega, \mathscr{F}, \mathbf{P}_{x} ; x \in I\right)$ with state space $(I, \mathscr{B}(I))$ let $\mathbf{F}^{0}=\left(\mathscr{F}_{t}^{0}\right)_{t \geqslant 0}$ denote the filtration generated by $X$. We set $\mathscr{F}_{\infty}^{0}:=\vee_{t \geqslant 0} \mathscr{F}_{t}^{0}$. We define $\mathscr{F}_{t}^{X}$ to be the completion of $\mathscr{F}_{t}^{0}$ in $\mathscr{F}$ with respect to $\left(\mathbf{P}_{\mu}\right)$ and write $\mathbf{F}^{X}=\left(\mathscr{F}_{t}^{X}\right)_{t \geqslant 0}$ as well as $\mathscr{F}_{\infty}^{X}:=\mathrm{V}_{t \geqslant 0} \mathscr{F}_{t}^{X}$. For a filtration $\mathbf{H}$, we denote the smallest right-continuous filtration containing $\mathbf{H}$ by $\mathbf{H}_{+}$.

D e fin ition 2.1. A continuous adapted process $X=\left(X_{t}\right)_{t \geqslant 0}$ on $\left(\Omega, \mathscr{F}, \mathbf{F}, \mathbf{P}_{x} ; x \in I\right)$ with state space $I$ is said to be a continuous strong Markov process if the following conditions are satisfied:

(i) $\mathbf{P}_{x}\left\{X_{0}=x\right\}=1$ for every $x \in I$.

(ii) $X$ admits shift operators, i.e., there exists a semigroup $\Theta=\left(\theta_{t}\right)_{0 \leqslant t \leqslant \infty}$ of operators $\theta_{t}: \Omega \rightarrow \Omega$ such that

(a) $X_{t+s}=X_{s} \circ \theta_{t}, 0 \leqslant t<\infty, s \geqslant 0$, holds a.s. and

(b) $X_{t} \circ \theta_{\infty}=X_{\infty}$ holds on $\left\{\omega: X_{\infty}(\omega)=\lim _{t \rightarrow \infty} X_{t}(\omega)\right.$ exists and is finite $\}, t \geqslant 0$, a.s.

(iii) For every non-negative $\mathscr{F}_{\infty}^{X}$-measurable random variable $Z$ we have

$$
\mathbf{E}_{x}\left(Z \circ \theta_{t} \mid \mathscr{F}_{t}\right)=\mathbf{E}_{X_{t}}(Z) \quad \mathbf{P}_{x^{-}} \text {a.s. for every } \quad x \in I .
$$

(iv) For every $\mathbf{F}_{+}^{X}$-stopping time $T$ and every non-negative $\mathscr{F}_{\infty}^{X}$-measurable random variable $Z$ we have

$$
\mathbf{E}_{x}\left(Z \circ \theta_{T} \mathbf{1}_{\{T<\infty\}} \mid \mathscr{F}_{T+}^{X}\right)=\mathbf{E}_{X_{T}}(Z) 1_{\{T<\infty\}} \quad \mathbf{P}_{x^{-a} \text { a.s. for every } x \in I .}
$$

By [6, Proposition I.8.12], we know $\mathbf{F}_{+}^{X}=\mathbf{F}^{X}$. Thus, since $\mathbf{F}^{X}$ is complete with respect to $\left(\mathbf{P}_{\mu}\right)_{\mu}$, the first entry time of $X$ into a Borel set $B \subseteq I$,

$$
D_{B}:=D_{B}(X):=\inf \left\{t \geqslant 0: X_{t} \in B\right\},
$$

where, by convention, $\inf \varnothing=\infty$, is an $\mathbf{F}^{X}$-stopping time (see [25, I.4.15]). We recall the classification of the points of the state space of a strong Markov process $X$. We introduce the abbreviations

$$
D_{x}:=D_{\{x\}}, \quad D_{x+}:=D_{(x, \infty) \cap I}, \quad D_{x-}:=D_{(-\infty, x) \cap I} .
$$

$D_{x}, D_{x+}$ and $D_{x-}$ are stopping times satisfying $\mathbf{P}_{x}\left\{D_{x \pm}=0\right\} \in\{0,1\}$ by the zero-one law of Blumenthal.

Defin ition 2.2 .

(i) A point $x \in I$ is called regular if $\mathbf{P}_{x}\left\{D_{x+}=0\right\}=\mathbf{P}_{x}\left\{D_{x-}=0\right\}=1$ holds. Otherwise, $x$ is called singular. We denote the set of regular points by $R$ and the set of singular points by $S$. 
(ii) If all interior points of $I$ are regular the process $X$ itself is called regular.

(iii) A point $x \in I$ is called left singular if $\mathbf{P}_{x}\left\{D_{x+}=0\right\}=0$, right singular if $\mathbf{P}_{x}\left\{D_{x-}=0\right\}=0$, absorbing if $\mathbf{P}_{x}\left\{D_{x+}=0\right\}=\mathbf{P}_{x}\left\{D_{x-}=0\right\}=$ 0 . We denote the set of left singular (respectively right singular) points by $K_{-}$(respectively $K_{+}$). For the set of absorbing points $K_{+} \cap K_{-}$we write $E$.

(iv) If $R=\bigcup_{n}\left(a_{n}, b_{n}\right)$ is the representation of the open set $R$ as the union of its components then we set

$$
\begin{aligned}
\breve{R} & :=\bigcup_{n}\left(a_{n}, b_{n}\right) \cup\left\{a_{n}: a_{n} \in K_{+}\right\} \cup\left\{b_{n}: b_{n} \in K_{-}\right\}, \\
S_{+} & :=K_{+} \backslash\left(\left\{a_{n}: a_{n} \in K_{+}\right\} \cup E\right), \\
S_{-} & :=K_{-} \backslash\left(\left\{b_{n}: b_{n} \in K_{-}\right\} \cup E\right) .
\end{aligned}
$$

The scale function and the speed measure are two characteristic quantities of Markov processes. Let us recall that a scale function is defined to be a continuous function $p: R \rightarrow \mathbf{R}$ that strictly increases on each component $(a, b)$ of $R$ and satisfies $\mathbf{P}_{x}\left\{D_{y}<D_{z}\right\}=(p(z)-p(x)) /(p(z)-p(y))$ (see [18]). In order to transform a strong Markov process into a strong Markov semimartingale we need a continuous and strictly increasing function $p: I \rightarrow \mathbf{R}$ such that the restriction $p_{\mid R}$ is a scale function. We therefore introduce the following hypothesis $(\mathbf{H})$ ensuring the existence of such a function. We remark that Theorem 3.1 relies on $(\mathbf{H})$.

Hypothesis (H). For every component $(a, b)$ of $R$ and every scale function $p$ of $X$ we assume

$$
\begin{aligned}
& p(a+):=\lim _{x \downarrow a} p(x)>-\infty, \quad \text { if } a \in I \text { and } \\
& p(b-):=\lim _{x \uparrow b} p(x)<+\infty, \quad \text { if } \quad b \in I .
\end{aligned}
$$

In other words, each end-point of a component $(a, b)$ is supposed to be attracting for $(a, b)$.

In particular, $(\mathbf{H})$ is satisfied for regular strong Markov processes.

Theorem 2.1 ([1, 5.21, 5.22]). Let $X$ be a continuous strong Markov process on $\left(\Omega, \mathscr{F}, \mathbf{F}, \mathbf{P}_{x} ; x \in I\right)$ satisfying hypothesis $(\mathbf{H})$. Then there exists a continuous and strictly increasing function $p: I \rightarrow \mathbf{R}$ such that the restriction $p_{\mid R}$ is a scale function of $X$ and

$$
p(x)=\int_{x_{0}}^{x} 1_{R}(y) d p(y)+\int_{x_{0}}^{x} 1_{I \backslash R}(y) d y
$$

holds for some interior point $x_{0}$ of $I$. The process $p(X)$ is a continuous strong Markov semimartingale on $\left(\Omega, \mathscr{F}, \mathbf{F}^{X}, \mathbf{P}_{q(x)} ; x \in p(I)\right)$, where $q$ denotes the inverse function of $p$.

In the following we call $p$ a scale function of $X$ according to $(H)$. 
For regular Markov processes the notion of speed measure is well-known (see [25, Section VII.3]). W. Schmidt [30] associated a speed measure to arbitrary continuous strong Markov processes (see [1, 5.13]). We use the speed measure only when dealing with strong Markov processes which admit a scale function according to $(\mathbf{H})$. Then the speed measure is uniquely determined by the following occupation times formula.

Theorem 2.2 (Occupation times formula [1, 5.27]). Let $X$ be a continuous strong Markov process on $\left(\Omega, \mathscr{F}, \mathbf{F}, \mathbf{P}_{x} ; x \in I\right)$ satisfying hypothesis (H) and $p: I \rightarrow \mathbf{R}$ a continuous and strictly increasing function whose restriction on $R$ is a scale function of $X$. Then there exists a unique measure $m$ on $(I \backslash E, \mathscr{B}(I \backslash E))$ such that, for every non-negative measurable function $f$ on $I$ with $f_{\mid E} \equiv 0$, we have

$$
\begin{aligned}
\int_{0}^{t} f\left(X_{s}\right) d s= & \int_{\breve{R} \backslash E} L^{p(X)}(t, p(y)) f(y) m(d y)+\int_{\left[X_{0}, \max _{s \leqslant t} X_{s}\right] \cap S_{+}} f(y) m(d y) \\
& +\int_{\left[\min _{s \leqslant t} X_{s}, X_{0}\right] \cap S_{-}} f(y) m(d y), \quad t \geqslant 0
\end{aligned}
$$

a.s.

Defin it i o $\mathrm{n} 2.3$. We call $m$ defined in Theorem 2.2 the speed measure of $X$ with respect to the scale function $p$.

We finally recall the notions of «zero energy», «zero quadratic variation» and «local Dirichlet process». We follow J. Bertoin [2] who introduced the class of so-called strong Dirichlet processes which is left invariant by time changes (see $[36,3.7]$ ).

D e f i n i t i o 2.4 . Let $(\Omega, \mathscr{F}, \mathbf{P})$ be a probability space with filtration $\mathbf{F}=\left(\mathscr{F}_{t}\right)_{t \geqslant 0}$.

(i) A finite sequence of $\mathbf{F}$-stopping times $\tau: 0=T_{0} \leqslant T_{1} \leqslant \cdots \leqslant T_{k}$ is called a random partition with respect to $\mathbf{F}$.

(ii) A sequence $\tau_{n}: 0=T_{0}^{n} \leqslant T_{1}^{n} \leqslant \cdots \leqslant T_{k_{n}}^{n}$ of random partitions is said to tend to the identity if
(a) $\lim _{n \rightarrow \infty} \sup _{l} T_{l}^{n} 1_{\left\{T_{l}^{n}<\infty\right\}}=\infty$ a.s.,
(b) $\left\|\tau_{n}\right\|:=\sup _{l}\left[\left(T_{l+1}^{n}-T_{l}^{n}\right) 1_{\left\{T_{l+1}^{n}<\infty\right\}}\right]$ converges to 0 a.s.

(iii) A continuous adapted process $Q$ has zero energy (in the strong sense) if

$$
\lim _{n \rightarrow \infty} \mathbf{E} \sum_{l: 0 \leqslant l \leqslant k, T_{l+1}<\infty}\left(X_{T_{l+1}}-X_{T_{l}}\right)^{2}=0
$$

holds for every sequence $\tau_{n}: 0=T_{0}^{n} \leqslant T_{1}^{n} \leqslant \cdots \leqslant T_{k_{n}}^{n}$ of random partitions tending to the identity.

(iv) A continuous adapted process $Q$ has zero quadratic variation (in the strong sense) if there exists a nondecreasing sequence of stopping times $\left(T_{n}\right)_{n \in \mathbf{N}}$ with $\lim _{n \rightarrow \infty} T_{n}=\infty$ a.s. such that, for each $n$, the stopped process $Q^{T_{n}}$ has zero energy in the strong sense. 
(v) A continuous strong Dirichlet process is defined to be a continuous adapted process admitting a decomposition $Y=Y_{0}+M+Q$, where $M$ is a continuous square integrable martingale with $M_{0}=0$ and $Q$ a continuous adapted process of zero energy in the strong sense with $Q_{0}=0$.

(vi) A continuous adapted process $Y$ is called a continuous strong local Dirichlet process up to the stopping time $T$ if there exists a nondecreasing sequence of stopping times $\left(T_{n}\right)_{n \in \mathbf{N}}$ with $\lim _{n \rightarrow \infty} T_{n}=T$ a.s. such that, for each $n$, the stopped process $Y^{T_{n}}$ is a strong Dirichlet process. We say $\left(T_{n}\right)_{n \in \mathbf{N}}$ reduces the strong local Dirichlet process. In the case $T=+\infty$ we briefly call $Y$ a continuous strong local Dirichlet process.

$\mathrm{R}$ e m a r k 2.1 (see $[36,3.4,3.6]$ ).

(i) A continuous process $Y$ is a strong local Dirichlet process if and only if it admits a decomposition $Y=Y_{0}+M+Q$, where $M$ is a continuous local martingale with $M_{0}=0$ and $Q$ a continuous adapted process of zero quadratic variation (in the strong sense) with $Q_{0}=0$. Such a decomposition is unique.

(ii) Every continuous process of finite variation has zero quadratic variation in the strong sense. Therefore, every continuous semimartingale is a continuous strong local Dirichlet process.

D e f i n i t i o n 2.5. A continuous process $Y=\left(Y_{t}\right)_{t \geqslant 0}$ on $(\Omega, \mathscr{F}, \mathbf{F}$, $\left.\mathbf{P}_{y} ; y \in I\right)$ with state space $I$ is said to be a continuous strong Markov local Dirichlet process if

(i) $Y$ is a continuous strong Markov process according to 2.1 and

(ii) there exist processes $M$ and $Q$ with $M_{0}=Q_{0}=0$ such that

(a) $Y=Y_{0}+M+Q \mathbf{P}_{y}$-a.s. for every $y \in I$,

(b) $M$ is a continuous local martingale with respect to $\mathbf{F}$ for every $\mathbf{P}_{y}, y \in I$, and

(c) $Q$ has zero quadratic variation in the strong sense with respect to $\mathbf{F}$ for every $\mathbf{P}_{y}, y \in I$.

\section{Strong Markov local Dirichlet processes as solutions to stochastic differential equations}

Throughout this section $X$ denotes a continuous strong Markov local Dirichlet process on $\left(\Omega, \mathscr{F}, \mathbf{F}, \mathbf{P}_{x} ; x \in I\right)$ with state space $I$. We write $R$, $K_{+}$and $K_{-}$for the set of its regular, right singular and left singular points, respectively, and define $S_{+}$and $S_{-}$as in Definition 2.2. We assume $X$ to satisfy $(\mathbf{H})$ and choose $p: I \rightarrow \mathbf{R}$ as in Theorem 2.1. By $[36,4.1(\mathrm{ii})]$, the inverse $q:=p^{-1}$ is absolutely continuous on $p(I)$. Let $m$ be the speed measure of $X$ with respect to $p$. We decompose $m$ into the sum of an absolutely continuous and a singular part with respect to the Lebesgue measure:

$$
m(A)=\int_{A} h(x) d x+n(A) \text { for all } A \in \mathscr{B}(I \backslash E)
$$


i.e., there exists a set $N \in \mathscr{B}(I \backslash E)$ with zero Lebesgue measure and $n((I \backslash E) \backslash N)=0$. Without loss of generality we may assume $h(x)=\infty$ for every $x \in N$. The starting point is the following result on the structure of continuous strong Markov local Dirichlet processes.

Theorem 3.1 [36, Theorem 4.4]. Let $X=\left(X_{t}\right)_{t \geqslant 0}$ be a continuous strong Markov local Dirichlet process on $\left(\Omega, \mathscr{F}, \mathbf{F}, \mathbf{P}_{x} ; x \in I\right)$ with the state space I satisfying $(\mathbf{H})$. Let $p: I \rightarrow \mathbf{R}$ be a scale function according to $(\mathbf{H})$. Then the part of zero quadratic variation $Q$ of $X$ has the form

$$
\begin{aligned}
Q_{t}= & d_{+}\left(X_{0}, \int_{0}^{t} 1_{S_{+}}\left(X_{s}\right) d s\right)-d_{-}\left(X_{0}, \int_{0}^{t} 1_{S_{-}}\left(X_{s}\right) d s\right) \\
& -\frac{1}{2} \int\left(p^{-1}\right)^{\prime}(y) 1_{p(R)}(y) d_{y} L^{p(X)}(t, y)
\end{aligned}
$$

a.s., where $d_{+}, d_{-}: I \times \mathbf{R}_{+} \rightarrow[0, \infty]$ are $\mathscr{B}^{u}(I) \otimes \mathscr{B}\left(\mathbf{R}_{+}\right)$-measurable functions such that, for every $x \in I, d_{ \pm}(x, \cdot)$ are continuous and nondecreasing.

The problem if the local martingale part $M(X)$ can be represented as a stochastic integral with respect to Brownian motion amounts to asking if $\langle M(X)\rangle$ is absolutely continuous with respect to Lebesgue measure. We need the following lemma of Motoo.

Lemma 3.1 (see $[8,3.56]$ ). Let $A$ be a non-negative increasing $\mathbf{F}^{X}$. adapted process satisfying the following conditions:

(a) $A_{0}=0$ a.s.

(b) $A_{t+s}=A_{s}+A_{t} \circ \theta_{s}$ a.s. for all $s, t \geqslant 0$,

(c) $d A_{t} \ll d t$ a.s.

Then there exists a non-negative $\mathscr{B}^{u}(I)$-measurable function $k: I \rightarrow[0, \infty)$ such that $A_{t}=\int_{0}^{t} k\left(X_{s}\right) d s, t \geqslant 0$, holds a.s.

In the following we assume $\langle M(X)\rangle$ to be a version of the quadratic variation of $X$ for every $\mathbf{P}_{x}, x \in \mathbf{R}$ (see [8, 3.39, 3.31]).

Theorem 3.2. Let $M(X)$ be the local martingale part of $X$ ard $q^{\prime} a$ density of $q: p(I) \rightarrow \mathbf{R}$. Then $\langle M(X)\rangle$ is absolutely continuous a.s. if and only if $h>0$ holds Lebesgue-a.e. on $\left\{x \in R: q^{\prime}(p(x))>0\right\}$. In that case we have

$$
\langle M(X)\rangle_{t}=\int_{0}^{t} \frac{q^{\prime} \circ p}{h}\left(X_{s}\right) 1_{R}\left(X_{s}\right) d s, \quad t \geqslant 0, \text { a.s. }
$$

P r o of. First we suppose $h>0$ a.s. on $\left\{x \in R: q^{\prime}(p(x))>0\right\}$. By the occupation times formula (Theorem 2.2) and the convention $\left(h_{\mid N}\right)^{-1}=0$, we then obtain

$$
\begin{aligned}
& \int_{0}^{t} h^{-1}\left(X_{s}\right) q^{\prime} \circ p\left(X_{s}\right) 1_{R}\left(X_{s}\right) d s \\
& \quad=\int L^{p(X)}(t, p(y)) h^{-1}(y) q^{\prime} \circ p(y) 1_{R}(y) m(d y)
\end{aligned}
$$




$$
\begin{aligned}
& =\int L^{p(X)}(t, p(y)) h^{-1}(y) q^{\prime} \circ p(y) 1_{R}(y) h(y) d y \\
& =\int L^{p(X)}(t, p(y)) q^{\prime} \circ p(y) 1_{R}(y) d y, \quad t \geqslant 0, \text { a.s. }
\end{aligned}
$$

By [34, Lemma 5.9], we know that $X$ admits a local time $L^{X}$ in the sense of an occupation times density satisfying

$$
q^{\prime}(p(y)) L^{p(X)}(t, p(y))=L^{X}(t, y), \quad y \in I .
$$

We conclude

$$
\begin{gathered}
\int_{0}^{t} h^{-1}\left(X_{s}\right) q^{\prime} \circ p\left(X_{s}\right) 1_{R}\left(X_{s}\right) d s=\int L^{X}(t, x) 1_{R}(x) d x \\
=\int_{0}^{t} 1_{R}\left(X_{s}\right) d\langle M(X)\rangle_{s}=\langle M(X)\rangle_{t}, \quad t \geqslant 0, \text { a.s. }
\end{gathered}
$$

exploiting the fact that $M(X)$ is supported on the set of regular points $R$ (see $[36,4.5])$.

Conversely, suppose that $\langle M(X)\rangle$ is absolutely continuous a.s. Since $\langle M(X)\rangle$ is increasing non-negative, $\mathbf{F}^{X}$-adapted and additive the Motoo Lemma 3.1 yields a non-negative $\mathscr{B}^{u}(I)$-measurable function $f$ such that

$$
\langle M(X)\rangle_{t}=\int_{0}^{t} f\left(X_{s}\right) d s, \quad t \geqslant 0
$$

holds a.s. Using the occupation times formula (Theorem 2.2) and again [34, Lemma 5.9] we get

$$
\begin{aligned}
\int & L^{p(X)}(t, p(y)) 1_{\{h=0\} \cap(R \backslash N)}(y) q^{\prime} \circ p(y) d y \\
& =\int L^{X}(t, x) 1_{\{h=0\} \cap(R \backslash N)}(x) d x=\int 1_{\{h=0\} \cap(R \backslash N)}\left(X_{s}\right) d\langle M(X)\rangle_{s} \\
& =\int 1_{\{h=0\} \cap(R \backslash N)}\left(X_{s}\right) f\left(X_{s}\right) d s \\
& =\int L^{p(X)}(t, p(y)) 1_{\{h=0\} \cap(R \backslash N)}(y) f(y) m(d y)=0, \quad t \geqslant 0 \text { a.s. }
\end{aligned}
$$

The last equality is valid by (5).

However, $p(X)$ is a continuous local martingale up to the stopping time $D_{R^{c}}(X)$ with respect to every $\mathbf{P}_{x}, x \in R$ (see $[1,2.20]$ ). Therefore, we have

$$
L^{p(X)}\left(t \wedge D_{R^{c}}(X), p(x)\right)>0
$$

for every $t \geqslant 0, \mathbf{P}_{x^{-}}$a.s. by $\left[12,2.24(\right.$ iii) $)$. As the local time of $p(X)^{D_{R^{c}(X)}}$ is a.s. continuous in the spatial parameter and $q^{\prime} \geqslant 0$ holds we realize by (6) 
that there exists an open neighbourhood $U(x)$ for every $x \in R$ such that $\mathbf{1}_{\{h=0\} \cap(R \backslash N)}(z) q^{\prime}(p(z))=0$ holds for Lebesgue-a.e. $z \in U(x)$. But this means $1_{\{h=0\} \cap(R \backslash N)}(z q) q^{\prime}(p(z))=0$ Lebesgue-a.e. Since $N$ is a null set it follows that $h>0$ holds Lebesgue-a.e. on $\left\{x \in R: q^{\prime}(p(x))>0\right\}$.

Since a continuous local martingale with absolutely continuous quadratic variation can be represented as a stochastic integral with respect to some Brownian motion on a possibly extended probability space (see [25, V.3.9], $[1,1.26]$ ), Theorems 3.1 and 3.2 now yield a criterion in terms of the scale function and the speed measure by which we can decide whether or not a given continuous strong Markov local Dirichlet process is a solution to a certain stochastic differential equation.

Theorem 3.3. Let $X$ be a continuous strong Markov local Dirichlet process on $\left(\Omega, \mathscr{F}, \mathbf{F}, \mathbb{P}_{x} ; x \in I\right), p: I \rightarrow \mathbf{R}$ a scale function according to $(\mathbf{H})$ and $m$ the corresponding speed measure. Then, on a possibly extended family of probability spaces, $X$ is a solution to the stochastic differential equation

$$
\begin{aligned}
X_{t}= & X_{0}+\int_{0}^{t} \sigma\left(X_{s}\right) d B_{s}+d_{+}\left(X_{0}, \int_{0}^{t} 1_{S_{+}}\left(X_{s}\right) d s\right) \\
& -d_{-}\left(X_{0}, \int_{0}^{t} 1_{S_{-}}\left(X_{s}\right) d s\right) \\
& -\frac{1}{2} \int q^{\prime}(y) 1_{p(R)}(y) d_{y} L^{p(X)}(t, y), \quad t \geqslant 0, \text { a.s. }
\end{aligned}
$$

where $\sigma: \mathbf{R} \rightarrow \mathbf{R}$ is measurable, $B$ is a Brownian motion and $d_{ \pm}: I \times$ $\mathbf{R}_{+} \rightarrow[0, \infty]$ are $\mathscr{B}^{u}(I) \otimes \mathscr{B}\left(\mathbf{R}_{+}\right)$-measurable functions such that, for every $x \in I, d_{ \pm}(x, \cdot)$ are continuous and nondecreasing, if and only if $h>0$ holds Lebesgue-a.e. on $\left\{x \in R: q^{\prime}(p(x))>0\right\}$.

In that case we can choose $\sigma:=\sqrt{q^{\prime} \circ p / h} 1_{R}$.

\section{Applications to Bessel processes}

We apply the results of Section 3 to Bessel processes of arbitrary dimension $d$. For $0<d<1$ we arrive at a new description of Bessel processes as solutions to a certain stochastic differential equation, and illustrate how it is related to known representations in terms of principal values. For Bessel processes of dimension $d \geqslant 1$ we recover the well-known stochastic differential equations by integration by parts.

\subsection{Bessel processes of dimension $0<d<1$.}

a) A Bessel process $X$ of dimension $0<d<1$ is known to be a continuous strong Markov process on a family of probability spaces $\left(\Omega, \mathscr{F}, \mathbf{F}^{X}, \mathbf{P}_{x}\right.$; $x \in[0, \infty))$ with state space $I=[0, \infty) . \quad R=(0, \infty)$ is its set of regular points, $K_{+}=\{0\}$ and $K_{-}=\varnothing$ are the sets of right singular and left singular points, respectively. So we have $S_{+}=S_{-}=\varnothing$. As a scale function 
according to $(\mathbf{H})$ we may choose $p(x)=x^{2-d}$. The corresponding speed measure is $m(d x)=-(d-2)^{-1} x^{d-1} d x$ (see [25, Chapter XI.1]).

Furthermore, $X$ is a continuous strong local Dirichlet process on every $\left(\Omega, \mathscr{F} / \mathbf{F}^{X}, \mathbf{P}_{x}\right), x \in[0, \infty)$, but not a semimartingale. This results from $[5$, Theorem II.1] and following discussion together with [4, Lemma 1.1]. Analogously to $[8,3.12]$, we can verify that $X$ is a continuous strong Markov local Dirichlet process admitting a universal decomposition in the sense of 2.7.

Theorem 3.3 now tells us that $X$ solves the equation

$$
\begin{aligned}
X_{t}= & X_{0}+\int_{0}^{t} \mathbf{1}_{(0, \infty)}\left(X_{s}\right) d B_{s} \\
& -\frac{1}{2(2-d)} \int y^{-(1-d) /(2-d)} \mathbf{1}_{(0, \infty)}(y) d_{y} L^{p(X)}(t, y),
\end{aligned}
$$

$t \geqslant 0$, a.s., where $B$ is a Brownian motion (possibly on an extended family of probability spaces). Because of $X \geqslant 0$ and

$$
\begin{aligned}
\left\langle\int_{0} 1_{\{0\}}\left(X_{s}\right) d B_{s}\right\rangle_{t} & =\int_{0}^{t} 1_{\{0\}}\left(X_{s}\right) d s \\
& =\int_{\mathbf{R}} L^{p(X)}(t, p(x)) 1_{\{0\}}(x) m(d x)=0
\end{aligned}
$$

$X$ turns out to be a solution to the stochastic differential equation

$$
X_{t}=X_{0}+B_{t}+\frac{1}{2(d-2)} \int y^{-(1-d) /(2-d)} 1_{(0, \infty)}(y) d_{y} L^{p(X)}(t, y), \quad t \geqslant 0, \text { a.s. }
$$

Note that the above Bouleau-Yor integral makes sense because the integrand is locally square integrable (see [34, 2.2]).

b) It is well known that the square $Y=X^{2}$ of a $d$-dimensional Bessel process is characterized as a unique strong solution to the stochastic differential equation

$$
Y_{t}=Y_{0}+2 \int_{0}^{t} \sqrt{Y_{s}} d B_{s}+d \cdot t, \quad t \geqslant 0
$$

where $B$ is a Brownian motion. We are now going to show that $F(x):=$ $x^{2}$ transforms a solution to equation (7) into a solution to the stochastic differential equation (8). Using the Itô formula of Russo and Vallois [27, Theorem 2.1] and integrating with respect to a Bouleau-Yor term thanks to [35, Theorem 2.1] we obtain

$$
\begin{aligned}
Y_{t} & =F\left(X_{t}\right)=Y_{0}+\int_{0}^{t} 2 X_{s} d^{-} X_{s}+[X]_{t} \\
& =Y_{0}+2 \int_{0}^{t} X_{s} d B_{s}
\end{aligned}
$$




$$
\begin{aligned}
& +\frac{1}{d-2} \int_{0}^{t} X_{s} d_{s}\left(\int y^{-(1-d) /(2-d)} 1_{(0, \infty)}(y) d_{y} L^{p(X)}(s, y)\right)+t \\
& =Y_{0}+2 \int_{0}^{t} \sqrt{Y_{s}} d B_{s} \\
& +\frac{1}{d-2} \int p^{-1}(y) y^{-(1-d) /(2-d)} 1_{(0, \infty)}(y) d_{y} L^{p(X)}(t, y) \\
& +\frac{1}{d-2} \int_{\mathbf{R}} y^{-(1-d) /(2-d)} 1_{(0, \infty)}(y) L^{p(X)}(t, y) d p^{-1}(y)+t \\
& =Y_{0}+2 \int_{0}^{t} \sqrt{Y_{s}} d B_{s}+\frac{1}{d-2} \int y^{d /(2-d)} 1_{(0, \infty)}(y) d_{y} L^{p(X)}(t, y) \\
& -\frac{1}{(d-2)^{2}} \int_{\mathbf{R}} y^{2(d-1) /(2-d)} 1_{(0, \infty)}(y) L^{p(X)}(t, y) d y+t
\end{aligned}
$$

Integrating by parts $[34,5.1]$ we conclude

$$
\begin{aligned}
Y_{t}= & Y_{0}+2 \int_{0}^{t} \sqrt{Y_{s}} d B_{s} \\
& +\frac{d-1}{(d-2)^{2}} \int_{\mathbf{R}} y^{2(d-1) /(2-d)} \mathbf{1}_{(0, \infty)}(y) L^{p(X)}(t, y) d y+t \\
= & Y_{0}+2 \int_{0}^{t} \sqrt{Y_{s}} d B_{s} \\
& +\frac{d-1}{(d-2)^{2}} \int_{\mathbf{R}} p(y)^{2(d-1) /(2-d)} \mathbf{1}_{(0, \infty)}(y) L^{p(X)}(t, p(y)) d p(y)+t \\
= & Y_{0}+2 \int_{0}^{t} \sqrt{Y_{s}} d B_{s} \\
& +\frac{d-1}{(d-2)^{2}} \int_{\mathbf{R}} y^{2(d-1)} \mathbf{1}_{(0, \infty)}(y) L^{p(X)}(t, p(y))(2-d) y^{1-d} d y+t \\
= & Y_{0}+2 \int_{0}^{t} \sqrt{Y_{s}} d B_{s}+(d-1) \int_{\mathbf{R}} \mathbf{1}_{(0, \infty)}(y) L^{p(X)}(t, p(y)) m(d y)+t .
\end{aligned}
$$

The occupation times formula (Theorem 2.2) now yields

$$
\begin{aligned}
Y_{t} & =Y_{0}+2 \int_{0}^{t} \sqrt{Y_{s}} d B_{s}+(d-1) \int_{0}^{t} \mathbf{1}_{(0, \infty)}\left(X_{s}\right) d s+t \\
& =Y_{0}+2 \int_{0}^{t} \sqrt{Y_{s}} d B_{s}+d \cdot t
\end{aligned}
$$

(c) In view of (a) and (b) we can characterize a Bessel process of dimension $0<d<1$ as a unique solution to the stochastic differential equation (7).

(d) It is well known that a Bessel process $X$ of dimension $0<d<1$ can be written as the sum of a Brownian motion and a principal value (see 
[25, XI.1.26], (5) and [37]). We shed light on the relations between the two representations by constructing $X$. In [5, Section V] and [10.1.3] we are told that a Bessel process $X$ of dimension $0<d<1$ is obtained by transforming linear Brownian motion $W$ with

$$
u(x):=(2-d)\left(x^{+}\right)^{1 /(2-d)}
$$

and then changing time with the right inverse $\left(\sigma_{t}\right)_{t \geqslant 0}$ of the increasing process

$$
A_{t}:=\int_{0}^{t}\left(W_{s}^{+}\right)^{-2(d-1) /(d-2)} d s, \quad t \geqslant 0: X_{t}:=u\left(W_{\sigma_{t}}\right),
$$

where $\sigma_{t}=\inf \left\{s \geqslant 0: A_{s}>t\right\}$. Since $u$ is absolutely continuous with locally square integrable density $u^{\prime}(x)=x^{-(1-\dot{d}) /(2-d)} \mathbf{1}_{(0, \infty)}(x)$ the Bouleau-Yor formula $[34,2.4]$ yields

$$
\begin{aligned}
X_{t}= & X_{0}+\int_{0}^{\sigma_{t}}\left(W_{s}{ }^{+}\right)^{-(1-d) /(2-d)} d W_{s} \\
& -\frac{1}{2} \int x^{-(1-d) /(2-d)} \mathbf{1}_{(0, \infty)}(x) d_{x} L^{W}\left(\sigma_{t}, x\right) \\
= & X_{0}+B_{t}-\frac{1}{2} \int x^{-(1-d) /(2-d)} \mathbf{1}_{(0, \infty)}(x) d_{x} L^{W}\left(\sigma_{t}, x\right),
\end{aligned}
$$

where

$$
B_{t}:=\int_{0}^{\sigma_{t}}\left(W_{s}+\right)^{-(1-d) /(2-d)} d W_{s}
$$

is seen to be a Brownian motion by P. Lévy's characterization theorem.

Since the Bouleau-Yor term can be looked upon as a continuous mapping defined on $L_{\text {loc }}^{2}(\mathbf{R})$ (see $\left.[34,2.4]\right)$ and $x^{-(1-d) /(2-d)} 1_{(\varepsilon, \infty)}(x)$ has finite variation, using integration by parts $[34,5.1]$ we calculate

$$
\begin{aligned}
\int x^{-(1-d) /(2-d)} \mathbf{1}_{(0, \infty)}(x) d_{x} L^{W}\left(\sigma_{t}, x\right) \\
=\lim _{\varepsilon \rightarrow 0} \int x^{-(1-d) /(2-d)} \mathbf{1}_{(\varepsilon, \infty)}(x) d_{x} L^{W}\left(\sigma_{t}, x\right) \\
=\lim _{\varepsilon \rightarrow 0}\left(\int_{\mathbf{R}} \frac{1-d}{2-d} x^{(2 d-3) /(2-d)} 1_{(\varepsilon, \infty)}(x) L^{W}\left(\sigma_{t}, x\right) d x\right. \\
\left.+\varepsilon^{-(1-d) /(2-d)} L^{W}\left(\sigma_{t}, \varepsilon\right)\right) .
\end{aligned}
$$

Since local time of Brownian motion is Hölder-continuous for each exponent less than $\frac{1}{2}$ and

$$
0<\frac{1-d}{2-d}<\frac{1}{2}, \quad \frac{2 d-3}{2-d}>-\frac{3}{2}
$$


hold, we obtain

$$
\begin{aligned}
& \int x^{-(1-d) /(2-d)} \mathbf{1}_{(0, \infty)}(x) d_{x} L^{W}\left(\sigma_{t}, x\right) \\
& =\lim _{\varepsilon \rightarrow 0}\left(\int_{\mathbf{R}} \frac{1-d}{2-d} x^{(2 d-3) /(2-d)} \mathbf{1}_{(\varepsilon, \infty)}(x) L^{W}\left(\sigma_{t}, x\right) d x\right. \\
& \left.\quad \quad \quad+\varepsilon^{-(1-d) /(2-d)} L^{W}\left(\sigma_{t}, 0\right)\right) \\
& =\lim _{\varepsilon \rightarrow 0} \frac{1-d}{2-d} \int_{\mathbf{R}} x^{(2 d-3) /(2-d)} \mathbf{1}_{(\varepsilon, \infty)}(x)\left(L^{W}\left(\sigma_{t}, x\right)-L^{W}\left(\sigma_{t}, 0\right)\right) d x \\
& =\frac{1-d}{2-d} \int_{\mathbf{R}} x^{(2 d-3) /(2-d)} \mathbf{1}_{(0, \infty)}(x)\left(L^{W}\left(\sigma_{t}, x\right)-L^{W}\left(\sigma_{t}, 0\right)\right) d x \\
& =\frac{1-d}{2-d} \int_{\mathbf{R}} x^{(2 d-3) /(2-d)} \mathbf{1}_{(0, \infty)}(x)\left(L^{W_{\sigma}^{+}}(t, x)-L^{W_{\sigma}^{+}}(t, 0)\right) d x \\
& =\frac{1-d}{2-d} \int_{\mathbf{R}} x^{(2 d-3) /(2-d)} \mathbf{1}_{(0, \infty)}(x)\left(L^{(2-d)^{d-2} p(X)}(t, x)\right. \\
& \left.\quad-L^{(2-d)^{d-2} p(X)}(t, 0)\right) d x,
\end{aligned}
$$

where $W_{\sigma}^{+}$denotes the positive part of the process obtained from $W$ by the time change $\sigma$. Using [25, VI.1.23] and substituting $x \leftrightarrow p(x)$ we finally arrive at

$$
\begin{aligned}
\int & x^{-(1-d) /(2-d)} \mathbf{1}_{(0, \infty)}(x) d_{x} L^{W}\left(\sigma_{t}, x\right) \\
& =\frac{1-d}{(2-d)^{2}} \int_{\mathbf{R}} x^{(2 d-3) /(2-d)} \mathbf{1}_{(0, \infty)}(x)\left(L^{p(X)}(t, x)-L^{p(X)}(t, 0)\right) d x \\
& =(1-d) \int_{\mathbf{R}} x^{d-2} \mathbf{1}_{(0, \infty)}(x) \frac{1}{2-d}\left(L^{p(X)}(t, p(x))-L^{p(X)}(t, 0)\right) d x \\
& =(1-d) \int_{\mathbf{R}} x^{-1} \mathbf{1}_{(0, \infty)}(x)\left(L^{p(X)}(t, p(x))-L^{p(X)}(t, 0)\right) m(d x) \\
= & :(1-d) \text { p.v. } \int_{0}^{t} \frac{1}{X_{s}} d s
\end{aligned}
$$

(see [37, p. 143]). Thus, from (9) we get the decomposition

$$
X_{t}=X_{0}+B_{t}+\frac{d-1}{2} \text { p.v. } \int_{0}^{t} \frac{1}{X_{s}} d s
$$

which can be found in $[25$, XI.1.26], $[37$, p. 123] or $[5$, p. 665, V.1]. On the other hand, (9) is equivalent to

$$
X_{t}=X_{0}+B_{t}-\frac{1}{2} \int x^{-(1-d) /(2-d)} \mathbf{1}_{(0, \infty)}(x) d_{x} L^{W_{\sigma}^{+}}(t, x)
$$




$$
=X_{0}+B_{t}+\frac{1}{2(d-2)} \int x^{-(1-d) /(2-d)} 1_{(0, \infty)}(x) d_{x} L^{p(X)}(t, x) .
$$

Thus we have established the stochastic differential equation (7) in an alternative way and have shown that the principal value in (12) represents a Bouleau-Yor integral:

$$
\text { p.v. } \int_{0}^{t} \frac{1}{X_{s}} d s=\frac{1}{(d-1)(d-2)} \int x^{-(1-d) /(2-d)} 1_{(0, \infty)}(x) d_{x} L^{p(X)}(t, x) .
$$

We finish by an informal discussion. Comparing the definition of the principal value and the occupation times formula we realize that the defining integral (11) is finite for no other reason than that the difference $\left(L^{p(X)}(t, p(x))-\right.$ $\left.L^{p(X)}(t, 0)\right)$ appears instead of $L^{p(X)}(t, p(x))$. Comparing (10) and (13) gives rise to consider the Bouleau-Yor integral as a tool of integration by parts which does not care about the constant (in the spatial parameter) $L^{p(X)}(t, 0)$.

4.2. Bessel processes of dimension $d \geqslant 1$. A Bessel process $X$ of dimension $d \geqslant 1$ is a continuous strong Markov semimartingale on $\left(\Omega, \mathscr{F}, \mathbf{F}^{X}\right.$, $\left.\mathbf{P}_{x} ; x \in[0, \infty)\right)$ with state space $I=[0, \infty)$ and the sets $R=(0, \infty)$, $K_{+}=\{0\}, K_{-}=\varnothing, S_{ \pm}=\varnothing$. As scale function $p$ according to $(\mathbf{H})$ and corresponding speed measure $m$ we can choose

$$
\begin{array}{ll}
p(x)=x^{2-d}, & m(d x)=-(d-2)^{-1} x^{d-1} d x ; \quad 1 \leqslant d<2, \\
p(x)=\ln (x), & m(d x)=x d x ; \quad d=2, \\
p(x)=-x^{2-d}, & m(d x)=(d-2)^{-1} x^{d-1} d x ; \quad d>2
\end{array}
$$

(see [25, Section XI.1]).

Theorem 3.3 yields

$$
\begin{aligned}
& X_{t}= X_{0}+\int_{0}^{t} \mathbf{1}_{(0, \infty)}\left(X_{s}\right) d B_{s} \\
&-\left\{\begin{array}{lr}
\frac{1}{2} \int(2-d)^{-1} y^{-(1-d) /(2-d)} 1_{(0, \infty)}(y) d_{y} L^{p(X)}(t, y), & 1 \leqslant d<2, \\
\frac{1}{2} \int \exp (y) d_{y} L^{p(X)}(t, y), & d=2, \\
\frac{1}{2} \int(d-2)^{-1}(-y)^{-(d-1) /(2-d)} 1_{(-\infty, 0)}(y) d_{y} L^{p(X)}(t, y), & d>2,
\end{array}\right.
\end{aligned}
$$

where $B$ is a Brownian motion. In the same way as above we verify that

$$
\int_{0}^{t} \mathbf{1}_{(0, \infty)}\left(X_{s}\right) d B_{s}=B_{t}
$$

always holds. 
If $d=1$ then we obtain

$$
\begin{aligned}
X_{t} & =X_{0}+B_{t}-\frac{1}{2} \int \mathbf{1}_{(0, \infty)}(x) d_{x} L^{p(X)}(t, x) \\
& =X_{0}+B_{t}+\frac{1}{2} L^{X}(t, 0)
\end{aligned}
$$

thus recovering the stochastic differential equation of Brownian motion reflected at 0 .

If $d>1$ then, using integration by parts [34,5.1] and

$$
L^{p(X)}(t, y)\left(p^{-1}\right)^{\prime}(y)=L^{X}\left(t, p^{-1}(y)\right)
$$

(see $[34,5.9]$ ) and observing $X \geqslant 0$, we recover the well known stochastic differential equation (see $[25$, pp. 427,431$]$ )

$$
X_{t}=X_{0}+B_{t}+\frac{d-1}{2} \int_{0}^{t} \frac{1}{X_{s}} d s
$$

We only give a proof for $d=2$, the other cases being analogous. We have

$$
\begin{aligned}
X_{t} & =X_{0}+B_{t}-\frac{1}{2} \int \exp (x) d_{x} L^{\ln (X)}(t, x) \\
& =X_{0}+B_{t}+\frac{1}{2} \int_{\mathbf{R}} L^{\ln (X)}(t, x) \exp (x) d x \\
& =X_{0}+B_{t}+\frac{1}{2} \int_{0}^{\infty} L^{\ln (X)}(t, \ln (x)) x d \ln (x) \\
& =X_{0}+B_{t}+\frac{1}{2} \int_{0}^{\infty} L^{X}(t, x) \frac{1}{x} d x \\
& =X_{0}+B_{t}+\frac{1}{2} \int_{0}^{t} \frac{1}{X_{s}} d s .
\end{aligned}
$$

Thus, for Bessel processes that are semimartingales Theorem 3.4 yields the known stochastic differential equations.

Looking at line (14) and informally integrating by parts we get

$$
X_{t}=X_{0}+B_{t}+\frac{1-d}{2} \int \ln (x) d_{x} L^{X}(t, x)
$$

In a forthcoming paper we will show that, indeed, this stochastic differential equation describes Bessel processes of arbitrary dimension. 


\section{REFERENCES}

1. Assing S., Schmidt W. Continuous Strong Markov Processes in Dimension 1. - A Stochastic Calculus Approach. Berlin etc.: Springer-Verlag, to appear in 1997.

2. Bertoin $J$. Temps locaux et intégration stochastique pour les processus de Dirichlet. - In: Séminaire de Probabilités, Lect. Notes Math., 1987, v. 1247, p. 191-205.

3. Bertoin J. Les processus de Dirichlet et tant qu' Espace de Banach. - Stochastics, 1988 , v. 18 , p. $155-168$.

4. Bertoin $J$. Sur une intégrale à $\alpha$-variation bornée. - Ann. Probab., 1989, v. 17, p. $1521-1535$.

5. Bertoin J. Complements on the Hilbert transform and the fractional derivative of Brownian local times. - J. Math. Kyoto Univ., 1990, v. 30, p. 651-670.

6. Blumenthal R. M., Getoor R. K. Markov Processes and Potential Theory. New York: Academic Press, 1968.

7. Bouleau $N$., Yor $M$. Sur la variation quadratique des temps locaux de certaines semimartingales. - C. R. Acad. Sci. Paris, Série I, 1981, v. 292, p. 491-494.

8. Cinlar E., Jacod J., Protter P., Sharpe M. J. Semimartingales and Markov Processes. - Z. Wahrscheinlichkeitstheor. verw. Geb., 1980, B. 54, S. 161-219.

9. Cinlar E., Jacod J. Representation of semimartingale Markoy processes in terms of Wiener processes and Poisson random measures. - In: Seminar on Stochastic Processes. Basel: Birkhäuser, 1981.

10. Engelbert $H$. J., Schmidt W. On one-dimensional stochastic differential equations with generalized drift. - In: Stochastic Differential Systems, Lecture Notes in Control and Information Sciences, 1985, v. 69, p. 143-155.

11. Engelbert $H$. J., Schmidt $W$. On solutions of one-dimensional stochastic differential equations without drift. - Z. Wahrscheinlichkeitstheor. verw. Geb., 1985, B. 68, S. 287-314.

12. Engelbert H. J., Schmidt W. Strong Markov continuous local martingales and solutions of one-dimensional stochastic differential equations. I; II; III. - Math. Nachr., 1989 , v. 143 , p. $167-184 ; 198.9$, v. 144 , p. $241-281 ; 1991$, v. 151 , p. 149-197.

13. Föllmer $H$. Calcul d'Itô sans probabilités. - In: Sém. Prob. XV, Lect. Notes Math., 1981 , v. 850 , p. $143-150$.

14. Föllmer H., Protter P., Shiryaev A. N. Quadratic covariation and an extension of Itô's formula. - Bernoulli, 1995, v. 1, p. 149-169.

15. Itô $K$. Differential equations determining Markov processes (in Japanese). - Zenkoku Shijō Sūgaku Danwakai, 1942, v. 1077, p. 1352-1400.

16. Itô $K$. On a stochastic integral equation. - Proc. Imp. Acad. Tokyo, 1946, v. 22, p. $32-35$.

17. Itô K. On stochastic differential equations. - Mem. Amer. Math. Soc.; 1951, v. 4, p. $1-51$.

18. Itô K., McKean H. P. Diffusion processes and their sample paths. Berlin etc.: Springer-Verlag, 1965.

19. Le Gall J. F. Applications du temps local aux équations différentielles stochastiques unidimensionnelles. - In: Sém. de Probabilités XVII, Lect. Notes Math., 1983, v. 986, p. $15-31$.

20. Le Gall $J$. F. One-dimensional stochastic differential equations involving local times of the unknown process. - In: Stochastic Analysis and Applications, Lect. Notes Math., 1984, v. 1095, p. 51-82.

21. Méléard $S$. Application du calcul stochastique à l'étude de processus de Markov réguliers sur $[0,1]$. - Stochastics, 1986, v. 19, p. 41-82.

22. Perkins E. Local time and pathwise uniqueness for stochastic differential equations. In: Séminaire de Probabilités XVI, Lect. Notes Math., 1982, v. 920, p. 201-208. 
23. Портеяко Н. И. Обобщениые диффузионные процессы. - Ккев: Наукова думка, 1982.

24. Protter P. Stochastic Integration and Differential Equations. Berlin, New York: Springer-Verlag, 1992.

25. Revuz D., Yor M. Continuous Martingales and Brownian Motion. Berlin, New York: Springer-Verlag, 1994.

26. Russo $F$., Vallois $P$. Forward, backward and symmetric stochastic integration. Probab. Theory Relat. Fields, 1993, v. 97, p. 403-421.

27. Russo $F$., Vallois $P$. The generalized covariation process and Itô formula. - Stochastic Proces. Appl., 1995, v. 59, p. 81-104.

28. Russo $F$., Vallois $P$. Itô formula for $C^{1}$-functions of semimartingales. - Probab. Theory Relat. Fields, 1996, v. 104, p. 27-41.

29. Schmidt $W$. On semimartingale diffusions and stochastic differential equations. Stoch. Stoch. Reports, 19990, v. 29, p. 404-427.

30. Schmidt $W$. Über streng Markovsche stetige Semimartingale. Habilitationsschrift, Friedrich-Schiller-Universität, Jena, 1991.

31. Скороход A. B. О непрерывных марковских процессах, являющихся мартингалами. - Теория вероятн. и ее примен., 1963, т. VIII, в. 4, с. 379-390.

32. Strook D. W., Varadhan S. R. S. Multidimensional Diffusion Processes. Berlin etc.: Springer-Verlag, 1979.

33. Wolf J. Zur stochastischen Analysis stetiger lokaler Dirichletprozesse. Dissertation, Friedrich-Schiller-Universität, Jena, 1997.

34. Wolf $J$. Transformations of semimartingales and local Dirichlet processes. - Stoch. Stoch. Reports, 1997, v. 62, p. 65-101.

35. Wolf J. An Itô formula for continuous local Dirichlet processes. - Stoch. Stoch. Reports, 1997, v. 62, p. 103-115.

36. Wolf $J$. On the decomposition of continuous strong Markov local Dirichlet processes. - Stochastic Proces. Appl., submitted.

37. Yor $M$. Some aspects of Brownian Motion, Part II: Some new martingal problems. Prépublication $N^{o}$ 125, Laboratoire de Probabilités, Université de Paris VI, 1992. 